\title{
Evidence of internal Bauschinger test in nanocomposite wires during in situ macroscopic tensile cycling under synchrotron beam
}

\author{
L. Thilly a) and P. O. Renault \\ Laboratoire de Métallurgie Physique, University of Poitiers, 86962 Futuroscope, France
}

S. Van Petegem, S. Brandstetter, B. Schmitt, and H. Van Swygenhoven

Paul Scherrer Institute, CH-5232 Villigen-PSI, Switzerland

\author{
V. Vidal and F. Lecouturier \\ Laboratoire National des Champs Magnétiques Pulsés, UPS-INSA-CNRS, 31400 Toulouse, France
}

(Received 23 March 2007; accepted 21 May 2007; published online 12 June 2007)

\begin{abstract}
In situ multiple tensile load-unload cycles under synchrotron radiation are performed on nanocomposite $\mathrm{Cu} / \mathrm{Nb}$ wires. The phase specific lattice strains and peak widths demonstrate the dynamics of the load-sharing mechanism where the fine $\mathrm{Cu}$ channels and the $\mathrm{Nb}$ nanotubes store elastic energy, leading to a continuous buildup of internal stress. The in situ technique reveals the details of the macroscopically observed Bauschinger effect. (C) 2007 American Institute of Physics.
\end{abstract}

[DOI: $10.1063 / 1.2748325$ ]

In 1881, Bauschinger evidenced that the mechanical response of a material can depend not only on the absolute value of the macroscopic applied stress but also on its direction, with a reverse (compressive) flow stress often lower than forward (tensile) flow stress. ${ }^{1}$ The observed asymmetry (so-called "Bauschinger effect") of the flow stress is usually ascribed to long-range effects (internal stresses due to dislocation-microstructure interactions) and/or short-range effects (directionality of mobile dislocations or annihilation with reverse strain). ${ }^{2,3}$ Because of the need to invert the direction of applied load during deformation, true Bauschinger tests cannot always be performed, as in the case for thin films or wires. For such sample geometries, special experimental setups are developed, with simple tensile loading-unloading tests. ${ }^{2,4}$

The strength of (nano)composites results from a complex interplay between the mechanical properties of the individual phases and the presence of interfaces. During deformation, internal stresses develop because of elastic mismatch between the phases resulting in the manifestation of the Bauschinger effect. ${ }^{5-7}$ When multiple tension-compression cycles are applied to the composite, the internal stresses will develop accordingly to the load transfer between the soft and the hard phase and lead to a rounding of the reverse loading curves and a decrease of the reverse yield stress, the extreme situation being that the soft phase yields in compression already during unloading of the applied tensile load. In this case, it is foreseen that the soft phase will undergo a true Bauschinger test during the macroscopic tensile load-unload cycle, despite it has never been directly observed.

Here we report on such a "built-in" Bauschinger test performed in situ at the materials beamline of the Swiss Light Source on a $\mathrm{Cu} / \mathrm{Nb}$ nanocomposite wire, where the use of the microstrip detector allows fast measurements of diffraction patterns over a $2 \theta$ range of $60^{\circ} .{ }^{8}$ The real-time resolved evolution of the lattice strain and peak width of the individual phases demonstrate the Bauschinger effect in the soft $\mathrm{Cu}$ phase during multiple load-unload tensile tests,

\footnotetext{
${ }^{a)}$ Electronic mail: ludovic.thilly@univ-poitiers.fr
}

meanwhile uncovering the details of the load-sharing mechanism.

The nanocomposite $\mathrm{Cu} / \mathrm{Nb}$ samples were obtained from a wire processed via severe plastic deformation to obtain a structure containing $85^{3} \mathrm{Nb}$ nanotubes embedded in a multiscale $\mathrm{Cu}$ matrix, as shown in Ref. 9. Nb nanotubes (thickness $t_{\mathrm{Nb}}=88 \mathrm{~nm}$; volume fraction $\left.X_{\mathrm{Nb}}=20.8 \%\right)$ are filled with $\mathrm{Cu}$, $\mathrm{Cu}-f$ (diameter $d_{\mathrm{Cu}-f}=130 \mathrm{~nm}$ ), separated by the finest $\mathrm{Cu}-0$ channels (width $d_{\mathrm{Cu}-0}=93 \mathrm{~nm}$ ); groups of $85 \mathrm{Nb}$ nanotubes are separated by $\mathrm{Cu}-1$ channels (width $d_{\mathrm{Cu}-1}=360 \mathrm{~nm}$ ); and groups of $85^{2}$ nanotubes are separated by $\mathrm{Cu}-2$ channels (width $d_{\mathrm{Cu}-2}=3.9 \mu \mathrm{m}$ ). Finally, the group of $85^{3}$ nanotubes is embedded in the external $\mathrm{Cu}$ jacket, $\mathrm{Cu}-3\left(d_{\mathrm{Cu}-3}=21.1 \mu \mathrm{m}\right)$. The wire has a diameter of $0.5 \mathrm{~mm}$. Transmission electron microscope (TEM) investigations revealed that the cross section of the thickness of $\mathrm{Nb}$ nanotubes is composed of one grain. These parallel grains are elongated along the wire axis with a $\langle 110\rangle$ axial texture. The $\mathrm{Cu}-f, \mathrm{Cu}-0$, and $\mathrm{Cu}-1$ channels (referred as "fine" $\mathrm{Cu}$ in the following) contain also one grain in cross section; these parallel grains are elongated along the wire axis with low dislocation density. The larger $\mathrm{Cu}-2$ and $\mathrm{Cu}-3$ channels (referred as "large" $\mathrm{Cu}$ ) are composed of grains from $200 \mathrm{~nm}$ to micrometer range, with high dislocation density. The fine nanochannels $(\mathrm{Cu}-f, \mathrm{Cu}-\mathrm{O}$, and $\mathrm{Cu}-1)$ correspond to $40 \%$ of the $\mathrm{Cu}$ matrix. The $\mathrm{Cu}$ matrix presents a double axial texture with $\langle 111\rangle$ and $\langle 200\rangle$ components. $^{9}$

The wires were locally thinned to obtain a reduced gauge section over $10 \mathrm{~mm}$ (see inset of Fig. 1) allowing to probe directly the nanocomposite interior of the wire that would be otherwise screened by the external $\mathrm{Cu}-3$ jacket. Multiple tensile loading-unloading tests up to fracture were conducted at room temperature with a strain rate of $10^{-5} \mathrm{~s}^{-1}$ and a $24.2 \mathrm{keV}$ x-ray beam scattering on crystallographic planes parallel to the tensile axis. Si powder was fixed on the sample surface for angular calibration. Peak profile analysis was performed using symmetric Pearson VII functions. As demonstrated in previous in situ experiments, ${ }^{10}$ the $(220)_{\mathrm{Cu}}$ reflection, arising from the $\mathrm{Cu}$ grains with $\langle 111\rangle$ or $\langle 200\rangle$ axial textures, is the superposition from two peaks, the first 


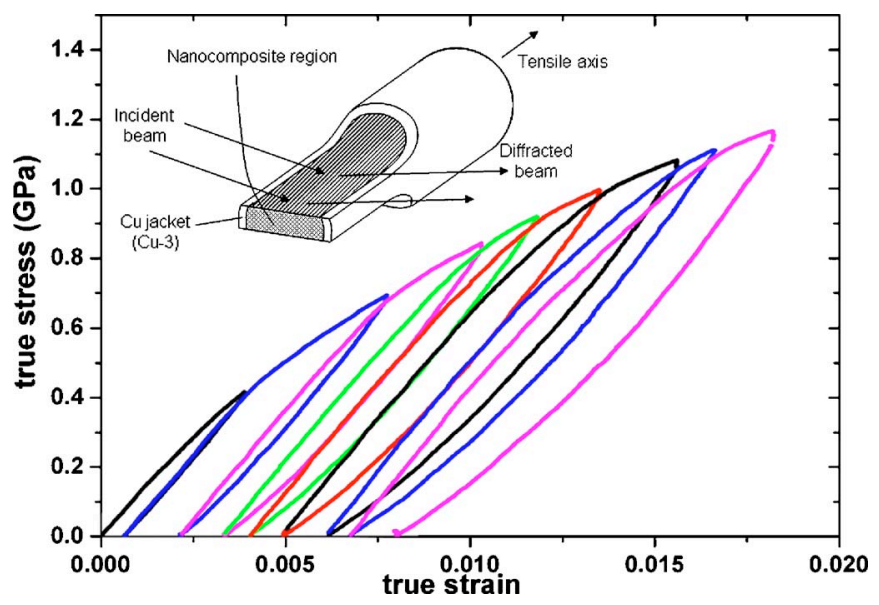

FIG. 1. (Color online) Macroscopic true-stress-true-strain curve of one tested wire. Inset: Schematic of one sample half with reduced section and beam scattering at crystallographic planes parallel to the tensile axis.

coming from the large- $\mathrm{Cu}$ channels ("large $\mathrm{Cu}$ peak"), the second from the fine- $\mathrm{Cu}$ channels ("fine $\mathrm{Cu}$ peak"). The $(220)_{\mathrm{Cu}}$ peak was fitted using two symmetric functions, with an integrated intensity ratio $I_{2} / I_{1}$ of 0.8 , in good agreement with the fine to large $\mathrm{Cu}$ volume fractions in the probed region, where most of the $\mathrm{Cu}-3$ jacket was removed.

Figure 1 presents the macroscopic true-stress-true-strain curve of one of the tested samples: the increasing hysteresis during tensile load-unload is the signature of large internal stresses that are built up. Figure 2(a) shows the applied stress versus run number (i.e., versus time since one run corresponds to a $30 \mathrm{~s}$ collection of $\mathrm{x}$ rays) with indication of the holding time in the loaded/unloaded states. It must be noted that during holding time the strain is kept constant. Figure 2(b) demonstrates the evolution of the (220) diffraction peak position of the large and fine $\mathrm{Cu}$ peaks versus run numbers and Fig. 2(c) the evolution of the (110) reflection in the $\mathrm{Nb}$ nanotubes. Already in the as-prepared state, the two $\mathrm{Cu}$ phases are in a different stress state: the fine $\mathrm{Cu}$ channels being in larger axial elastic compression than the large $\mathrm{Cu}$ channels since $2 \theta_{220}($ fine $\mathrm{Cu})<2 \theta_{220}($ large $\mathrm{Cu})$ $<2 \theta_{220}($ annealed $\mathrm{Cu})=23.12^{\circ}$. The $\mathrm{Nb}$ nanotubes are in

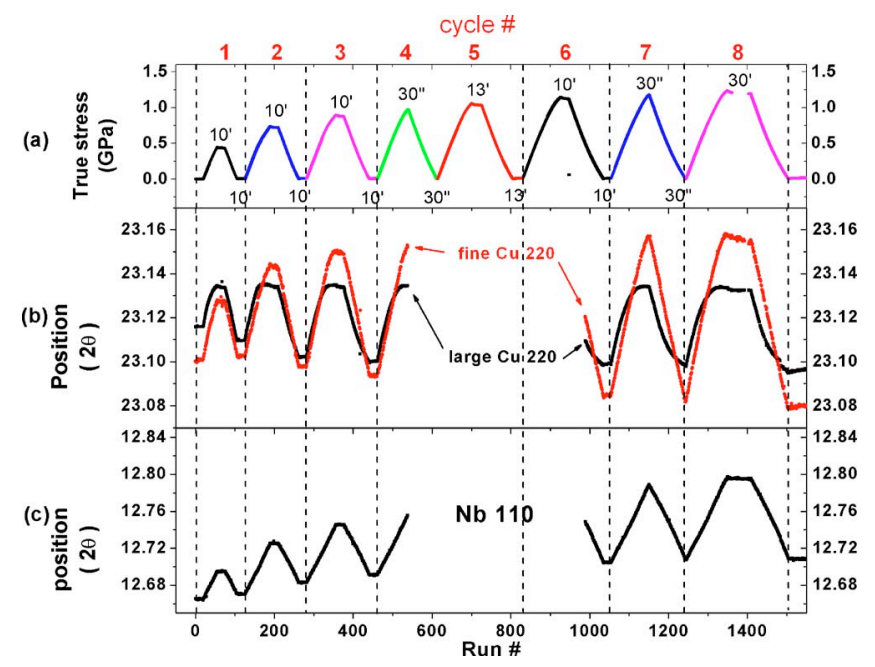

FIG. 2. (Color online) Evolution vs run numbers (i.e., time) and loadingunloading cycles of (a) true stress, with various holding times at loaded/ unloaded states; (b) position of (220) large- $\mathrm{Cu}$ and fine-Cu peaks; and (c) position of (110) $\mathrm{Nb}$ peak.

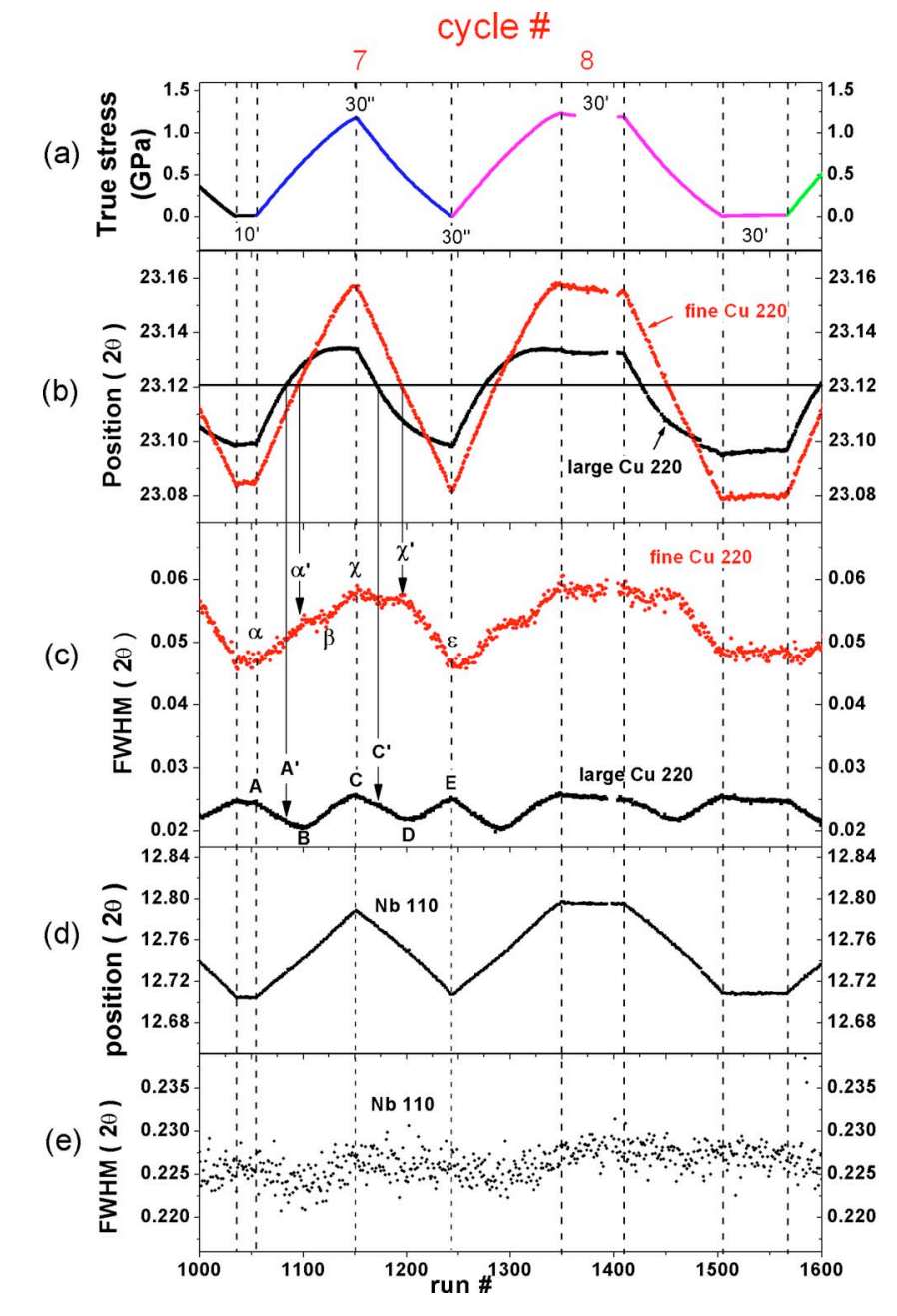

FIG. 3. (Color online) Evolution vs run numbers during cycles 7 and 8 of (a) true stress; (b) and (c) position and FWHM of (220) large-Cu and fine$\mathrm{Cu}$ peaks; and (d) and (e) position and FWHM of (110) Nb peak.

axial elastic tension $\left[2 \theta_{110}(\mathrm{Nb})>2 \theta_{110}(\right.$ annealed $\mathrm{Nb})$ $=12.60^{\circ} \mathrm{J}$. Note that both equilibrium $2 \theta$ values were determined by neutron scattering on annealed $\mathrm{Cu}$ and $\mathrm{Nb}$ samples. Applying tensile load, the two $\mathrm{Cu}$ phases respond differently: the shift of the peak position of the large $\mathrm{Cu}$ stabilizes upon loading [Fig. 2(b)], evidencing a pronounced plasticity regime whereas the fine-Cu peak exhibits only a slight deviation from linear behavior at highest stress. The $\mathrm{Nb}$ nanotubes remain in the elastic regime, as demonstrated by the linear behavior of the peak position versus time [Fig. 2(c)] and versus stress (not shown here). The material is therefore composed of three phases with distinct elastic-plastic behavior, a situation that favors the development of internal stresses during codeformation, as evidenced by the gradual change of the peak positions at each unloaded state: both the axial compression of $\mathrm{Cu}$ channels and the axial tension of $\mathrm{Nb}$ nanotubes increase after each cycle.

Figure 3 focuses on cycles 7 and 8 . The evolution of peak position and full width at half maximum (FWHM) versus run numbers for the large and fine (220) $\mathrm{Cu}$ peaks is given, respectively, in Figs. 3(b) and 3(c) and the (110) reflection in the $\mathrm{Nb}$ nanotubes is provided in Figs. 3(d) and 3(e). Having in mind that the broadening of diffraction peaks is induced by the presence of inhomogeneous strains such as the ones introduced by dislocations, the evolution of FWHM can be related to the peak position evolution. In Fig. 3(c), 
tensile load is applied from A (respectively $\alpha$ ) to C (respectively $\chi$ ), where the starain is kept constant during $30 \mathrm{~s}$, following an unloading with the same strain rate arriving in the macroscopic unloaded state E (respectively $\varepsilon$ ). When applying tensile load at $\mathrm{A}$, the FWHM of the large $\mathrm{Cu}$ peak decreases first, reaching a local minimum (B), followed by an increase until the maximum load is attained in C. During macroscopic unloading, the FWHM first decreases from C to $\mathrm{D}$ followed by an increasing phase from $\mathrm{D}$ to $\mathrm{E}$. Note that the large $\mathrm{Cu}$ phase is unloaded at $\mathrm{A}^{\prime} \mathrm{C}^{\prime}$. The evolution of FWHM can be interpreted as the following: during macroscopic loading from $\mathrm{A}$ to $\mathrm{A}^{\prime}$, the large $\mathrm{Cu}$ channels, initially in axial compression, are unloaded and FWHM decreases. Then from $\mathrm{A}^{\prime}$ to $\mathrm{C}$, the large $\mathrm{Cu}$ channels are put into tension but owing to a low yield stress they soon enter in a plastic regime and FWHM increases at $\mathrm{B}$. When the specimen is macroscopically unloaded from $\mathrm{C}$ to $\mathrm{C}^{\prime}$, the large $\mathrm{Cu}$ channels are unloaded and FWHM decreases. Since the macroscopic stress is higher in the whole sample compared to the large $\mathrm{Cu}$ channels, when the specimen is further unloaded from $\mathrm{C}^{\prime}$ to $\mathrm{E}$, these channels are put into compression and enter in a plastic regime because of low yield stress: FWHM increases again at $\mathrm{D}$.

The FWHM for the (220) fine $\mathrm{Cu}$ peak behaves differently: upon loading from macroscopic unloaded state $\alpha$, it first increases linearly until the unloaded state is reached, indicated by $\alpha^{\prime}$ where it suddenly levels off. As long as the peak position of the fine $\mathrm{Cu}$ phase increases linearly, the FWHM stays approximately constant. When the peak position starts deviating from linear behavior (indicated by $\beta$ ) the FWHM starts increasing again until the maximum macroscopic tensile load is reached at $\chi$. During unloading, FWHM decreases very slowly until the unloaded state is reached $\left(\chi^{\prime}\right)$, then rapidly until macroscopic unloaded state $(\varepsilon)$. The above mentioned trends are confirmed in cycle 8 , with the difference that here the holding time was $30 \mathrm{~min}$. Previous in situ TEM deformation studies have shown that the fine $\mathrm{Cu}$ deforms by nucleation of noncorrelated single dislocation loops nucleated at the $\mathrm{Cu} / \mathrm{Nb}$ interfaces and expanding until interaction with a neighboring interface. ${ }^{11}$ Such a mechanism can explain the particular behavior of the FWHM of the fine $\mathrm{Cu}$ channels [Fig. 3(c)]. At the highest compressive state $(\alpha)$, most of the dislocations (nucleated in previous cycles) are pushed back into the interfaces. During macroscopic tensile loading, the fine $\mathrm{Cu}$ first unloads until $\alpha^{\prime}$ and the dislocations return to an equilibrium configuration, resulting in an increase of the FWHM. Then when the fine $\mathrm{Cu}$ phase is elastically loaded, the FWHM stays approximately constant ( $\alpha^{\prime}$ to $\beta$ ) and only when the plastic regime is reached previous and fresh dislocations expand further between the $\mathrm{Cu}-\mathrm{Nb}$ interfaces (from $\beta$ to $\chi$ ). That the FWHM does not decrease substantially upon tensile unloading ( $\chi$ to $\left.\chi^{\prime}\right)$ suggests the necessity of a force to unpin the dislocations from $\mathrm{Cu}-\mathrm{Nb}$ interfaces. Note that a similar "delay" is observed at the beginning of cycle 8 and could not be distinguished in cycle 7 .

Finally, for the $\mathrm{Nb}$ nanotubes which remain in the elastic regime in axial tension, a slight increase of FWHM after each loading-unloading cycle is observed [Fig. 3(e)] suggesting a gradual increase of root mean square strain induced by

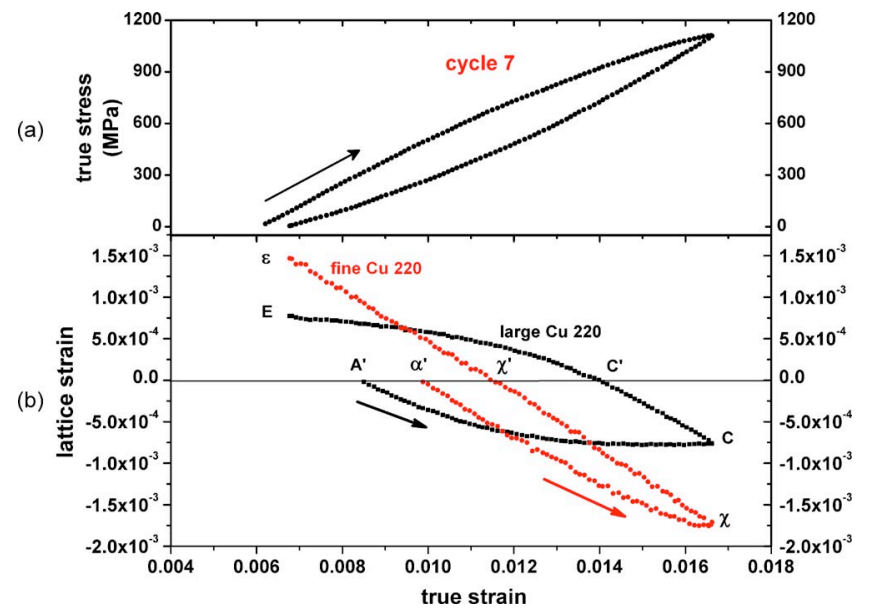

FIG. 4. (Color online) Evolution vs true strain during cycle 7 of (a) true stress; (b) position of (220) large- $\mathrm{Cu}$ and fine-Cu peaks. The $\mathrm{Cu}$ channels are subjected to an internal Bauschinger test during each cycle (negative longitudinal lattice strain during tension and positive during compression).

the load transfer from the plastifying $\mathrm{Cu}$ channels onto the elastic $\mathrm{Nb}$ nanotubes. ${ }^{10}$

Figure 4 shows the true stress [Fig. 4(a)], the lattice strains [Fig. 4(b)], and FWHM derived of the (220) large and fine $\mathrm{Cu}$ diffraction now as function of the macroscopic tensile strain during cycle 7 . The plots show that (1) during one tensile loading-unloading cycle, the $\mathrm{Cu}$ channels are subjected to a true Bauschinger test, i.e., a tension-compression cycle, and (2) the "Bauschinger" hysteresis visible in the macroscopic stress-strain curve results from the reverse yielding of the large-Cu phase evidenced in the strong rounding upon unloading [Fig. 4(b)] and the increase in FWHM at $\mathrm{D}$ [Fig. 3(c)]. The unloading of the fine $\mathrm{Cu}$ channels is, however, purely elastic, which can be explained by the higher value of the yield stress related to the size of the fine $\mathrm{Cu}$ channels and the particular dislocation mechanism associated.

In summary, the above results demonstrate the power of in situ techniques in revealing the dynamics of the phase specific lattice strains and deformation mechanisms reflected in peak broadening, shedding light on the origin of the macroscopically observed Bauschinger effect. The technique allows exploring the physical origins of the Bauschinger effect by performing simple tensile load-unload experiments meanwhile making advantage of the naturally built-in Bauschinger test in a nanocomposite.

${ }^{1}$ J. Bauschinger, Civiling N.F. 27, 289 (1881).

${ }^{2}$ Y. Xiang and J. J. Vlassak, Acta Mater. 54, 5449 (2006).

${ }^{3}$ Y. Brechet and P. Jarry, J. Phys. III 1, 1985 (1991).

${ }^{4}$ C. Sinclair, G. Saada, and J. Embury, Philos. Mag. 86, 4081 (2006).

${ }^{5}$ M. Ashby, Philos. Mag. 21, 399 (1970).

${ }^{6} \mathrm{R}$. Asaro, Acta Metall. 23, 271 (1975).

${ }^{7}$ J. Eshelby, Proc. R. Soc. London, Ser. A 241, 376 (1957).

${ }^{8}$ H. Van Swygenhoven, B. Schmitt, P. M. Derlet, S. Van Petegem, A. Cervellino, Z. Budrovic, S. Brandstetter, A. Bollhalder, and M. Schild, Rev. Sci. Instrum. 77, 013902 (2006).

${ }^{9}$ V. Vidal, L. Thilly, F. Lecouturier, and P. O. Renault, Scr. Mater. 57, 245 (2007).

${ }^{10}$ L. Thilly, V. Vidal, S. Van Petegem, U. Stuhr, F. Lecouturier, P. O. Renault, and H. Van Swygenhoven, Appl. Phys. Lett. 88, 191906 (2006).

${ }^{11}$ L. Thilly, O. Ludwig, M. Véron, F. Lecouturier, J. P. Peyrade, and S. Askénazy, Philos. Mag. A 82, 925 (2002). 